\title{
Binding Quantum Dots to Silk Biomaterials for Optical Sensing
}

\author{
Disi Lu, ${ }^{1}$ Zhaozhu Zheng, ${ }^{2}$ Shaozhe Guo, ${ }^{2}$ Cheng Wang, \\ David L. Kaplan, ${ }^{3}$ and Xiaoqin Wang ${ }^{2}$ \\ ${ }^{1}$ College of Chemical Engineering and Material Chemistry, Heilongjiang University, Harbin 150086, China \\ ${ }^{2}$ National Engineering Laboratory for Modern Silk, Soochow University, Suzhou 215123, China \\ ${ }^{3}$ Department of Biomedical Engineering, Tufts University, Medford, MA 02155, USA \\ Correspondence should be addressed to Xiaoqin Wang; xiaoqin.wang@tufts.edu
}

Received 18 May 2015; Accepted 26 July 2015

Academic Editor: Won-Gun Koh

Copyright (C) 2015 Disi Lu et al. This is an open access article distributed under the Creative Commons Attribution License, which permits unrestricted use, distribution, and reproduction in any medium, provided the original work is properly cited.

\begin{abstract}
Quantum dots (QDs), have great potential for fabricating optical sensing devices and imaging biomaterial degradation in vivo. In the present study, 2-mercaptoethylamine- (MEA-) and mercaptopropionic acid- (MPA-) capped CdTe-QDs were physically incorporated in silk films that contained a high content $(>30 \%)$ of crystalline beta-sheet structure. The beta-sheets were induced by the addition of glycerol, water annealing, glycerol/annealing, or treatment with methanol. Incorporation of QDs did not influence the formation of beta-sheets. When the films were extracted with water, most QDs remained associated with the silk, based on the retention of photoluminescence in the silk films and negligible photoluminescence in the extracts. Compared to the solution state, photoluminescence intensity significantly decreased for MEA-QDs but not for MPA-QDs in the silk films, while the emission maximum blue shifted $(\approx 4 \mathrm{~nm})$ slightly for both. Further film digestion using protease XIV, alpha-chymotrypsin, and the combination of the two proteases suggested that QDs may be bound to the silk beta-sheet regions but not the amorphous regions. QDs photoluminescence in silk films was quenched when the concentration of hydrogen peroxide $\left(\mathrm{H}_{2} \mathrm{O}_{2}\right)$ was above $0.2-0.3 \mathrm{mM}$, indicating the QDs-incorporated silk films can be used to report oxidation potential in solution.
\end{abstract}

\section{Introduction}

Quantum dot (QD) is a type of synthetic nanocrystal composed of an inorganic core and an organic outer layer of surfactant molecules (ligands). Compared with organic dyes, QDs possess greater brightness, better stability with respect to photobleaching, and narrower spectral line widths [1], thus having potential for optoelectronic and photovoltaic devices, optical amplifier media, and bioimaging [2]. QDs have been used as photoluminescence probes to detect many analytes, such as hydrogen ions $(\mathrm{pH})$, metal ions, organic compounds, and biomolecules in a growing range of chemical and bioanalytical applications [3-5]. ODs have also been used in glucose oxidase (GOX-) based glucose monitoring; for example, photoluminescence of thioglycolic acid- (TGA-) capped CdTe-QDs was quenched in response to the change of $\mathrm{H}_{2} \mathrm{O}_{2}$ and $\mathrm{pH}[3,6,7]$. The in vivo uses of QDs have been hindered mainly due to toxicity and potential risks to the environment [8]. Various modifications have been employed to improve the stability and safety of QDs for in vivo applications, including surface modifications, coatings, and encapsulation in carriers [9].

Assembling QDs on solid surfaces with minimal influence of photoluminescence is critical for their integration with solid-state devices. Subtle interactions between the surface atoms of QDs and surrounding molecules can significantly influence the photoluminescence characteristics of QDs. Several fabrication techniques are used to make ultrathin organized nanocomposite films for the incorporation of QDs, including spin casting, Langmuir-Blodgett (LB) deposition, and layer-by-layer (LbL) assembly [2, 10, 11]. However, there are challenges to maintain the photoluminescence stability of QDs in the solid state: (i) the QD photoluminescence quantum yields can be significantly reduced via solvent transfer steps usually involved in fabrication procedures. (ii) QD photoluminescence quantum yields can rapidly deteriorate 
upon continuous excitation in solid state as a function of moisture, oxygen, heat, and light, worse than in solution phase where an excess of surface ligands passivate the QD surface. (iii) Optical spectra can be red-shifted or blue-shifted due to QD aggregation or degradation, respectively, during material solidification process [12]. Aside from coating with amphiphilic molecules, such as phospholipids, polymers, and saccharides [13-15], one effective method to stabilize QDs is to incorporate them into an appropriate host matrix such as organic polymers, carbon nanomaterials, and inorganic materials through in situ nanocrystal growth, electrostatic interactions, and cross-linking processes [16-18]. To achieve these improvements, the original procedures for material synthesis need to be modified or reoptimized, which is not practical in most applications.

Silks spun by spiders and silkworms represent the strongest and toughest natural fibers known and offer unlimited opportunities for functionalization, processing, and biological integration [19]. Silk fibroin protein isolated from Bombyx mori silkworm cocoons can be used directly for surface coatings or the fabrication of various biomaterials (films, hydrogels, sponges, fibers, and particles), thus holding promise in biomedical applications ranging from tissue engineering to controlled drug delivery due to the unique structural and biological properties [19]. Long-term stabilization and sustained release of bioactive molecules from silk biomaterials have been well documented [20, 21]. Other advantages of using silk as biomaterials include solvent-free processing and sterilization by autoclaving or gamma irradiation [22]. Silk films have been widely studied and can be prepared by drying silk solution in the air and further treated by water vapor or alcohol to increase content of thermodynamically stable betasheets (over $50 \%$ of total secondary structures of fibroin) and thus crystallinity [22]. No chemical cross-linking reactions or postprocessing cross-linking is needed during these processes. Through fine-tuning beta-sheet content, such as timeand temperature-dependent water annealing, mechanically robust films with tunable thicknesses (from below ten nanometers to hundreds of micrometers or more), high surface quality (surface roughness about $5 \mathrm{~nm}$ RMS) and transparency (over $90 \%$ transmission across the visible range) can be obtained, which are suitable for optical and electronic substrate uses [23].

In a previous study we showed that 3-mercaptopropionic acid- (MPA-) coated CdTe-QDs could be incorporated in silk hydrogels and microspheres with minimal impact on photoluminescence properties and silk structures (to be published). In the present study, the interaction between two different types of QDs (MPA-capped and 2-mercaptoethylamine(MEA-) capped) and silk was further investigated by monitoring photoluminescence changes under various conditions, such as different film treatments, proteases digestion, and oxygen peroxide $\left(\mathrm{H}_{2} \mathrm{O}_{2}\right)$ quenching. The results elucidated the mechanism of QDs binding to silk and demonstrated the potential of using QDs-bound silk biomaterials in biosensing but also for tracking the in vivo degradation of silk biomaterials.

\section{Materials and Methods}

2.1. Materials. Partially degummed silk fibers were purchased from Xiehe Silk Corporation Ltd. (Hangzhou, China). Lithium bromide ( $\mathrm{LiBr}$ ) was purchased from Aladdin (Shanghai, China). $\mathrm{Cd}\left(\mathrm{ClO}_{4}\right)_{2} \cdot 6 \mathrm{H}_{2} \mathrm{O}, \mathrm{Al}_{2} \mathrm{Te}_{3}$ powders, 2mercaptoethylamine (MEA), and mercaptopropionic acid (MPA) were purchased from Alfa Aesar (MA, USA). Protease $\mathrm{XIV}$ and alpha-chymotrypsin were purchased from SigmaAldrich. Analytical grade hydrogen peroxide $\left(\mathrm{H}_{2} \mathrm{O}_{2}\right)$ and other chemical reagents were all purchased from Sinopharm Chemical Reagent Corporation Ltd. (Shanghai, China).

\subsection{Methods}

2.2.1. Synthesis of CdTe-QDs. The CdTe-QDs were prepared by colloidal methods as reported in the literature [24, 25]. Briefly, $0.985 \mathrm{~g}(2.35 \mathrm{mmol})$ of $\mathrm{Cd}\left(\mathrm{ClO}_{4}\right)_{2} \cdot 6 \mathrm{H}_{2} \mathrm{O}$ was added to $125 \mathrm{~mL}$ water. To this, $5.7 \mathrm{mmol} \mathrm{MPA}$ or MEA was added. The $\mathrm{pH}$ of the above solution was adjusted to 11.2 or 5.6 and purged with $\mathrm{N}_{2}$. The tellurium precursor $\mathrm{H}_{2} \mathrm{Te}$ gas was prepared by reaction of $0.2 \mathrm{~g}(0.46 \mathrm{mmol})$ of $\mathrm{Al}_{2} \mathrm{Te}_{3}$ lumps with $15-20 \mathrm{~mL}$ of $0.5 \mathrm{M} \mathrm{H}_{2} \mathrm{SO}_{4}$ under $\mathrm{N}_{2}$ atmosphere. $\mathrm{H}_{2}$ Te gas was passed through the solution together with a slow nitrogen flow for $\sim 20 \mathrm{~min}$. CdTe precursors were formed at this stage as seen by a change of the solution color. The precursors were converted to $\mathrm{CdTe}$ nanocrystals by refluxing the reaction mixture at $100^{\circ} \mathrm{C}$. The size of the CdTe-QDs grew further with time and was controlled by the duration of reflux, which was monitored by photoluminescence spectroscopy.

2.2.2. Purification of Silk Fibroin. Silk fibroin was purified by degumming in sodium carbonate solution and dissolving in lithium bromide solution as reported in the literature [22]. Briefly, silk fibers were boiled in $0.02 \mathrm{M}$ sodium carbonate solution for $30 \mathrm{~min}$, rinsed with ultrapure water three times, drained, and dried in a fume hood overnight. The dried fibers were dissolved in $9.3 \mathrm{M}$ lithium bromide solution at $60^{\circ} \mathrm{C}$ for $4 \mathrm{~h}$ to obtain a concentration of $20 \%$. The solution was dialyzed against pure water for $30 \mathrm{~h}$ to remove the lithium bromide and centrifuged to remove insoluble fibrous debris. The concentration of purified silk was about 8-9 wt.\%.

2.2.3. Incorporation of QDs in Silk Films. QDs were incorporated in silk films that were prepared following standard protocols [22]. Briefly, synthesized QDs (31.5 mM) were added to $4 \%$ silk solution. The mixed solution was aliquoted into a 24-well plate with $300 \mu \mathrm{L}$ for each well, and the plate was placed in a fume hood overnight for drying. The mass of silk was approximately $12 \mathrm{mg}$ in each film. The amount of MPAQDs loaded was $0,9.75,19.5,39,52$, and $78 \mu \mathrm{mol}$, and the amount of MEA-QDs was $0,15.75,31.5,63,84$, and $126 \mu \mathrm{mol}$. Silk and silk/QDs films in each well were treated as follows: (1) silk solution was supplemented with $1.3 \%$ (w/v) glycerol prior film casting; (2) as-cast silk films were vacuumed overnight in a desiccator with a water tank at the bottom (waterannealing); (3) silk/glycerol films were water annealed as described above (glycerol and water-annealing); (4) films were immersed in $300 \mu \mathrm{L}$ methanol for $1 \mathrm{~h}$ and then redried in 

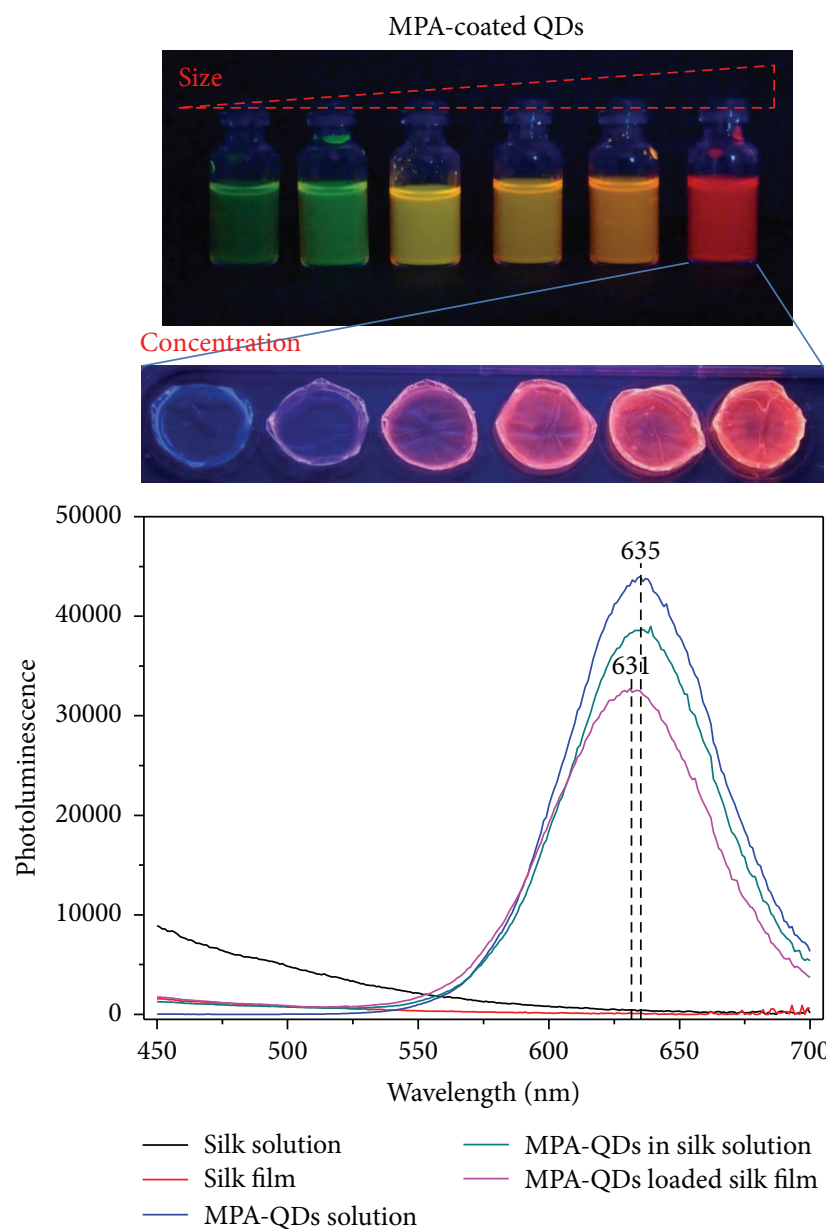

(a)
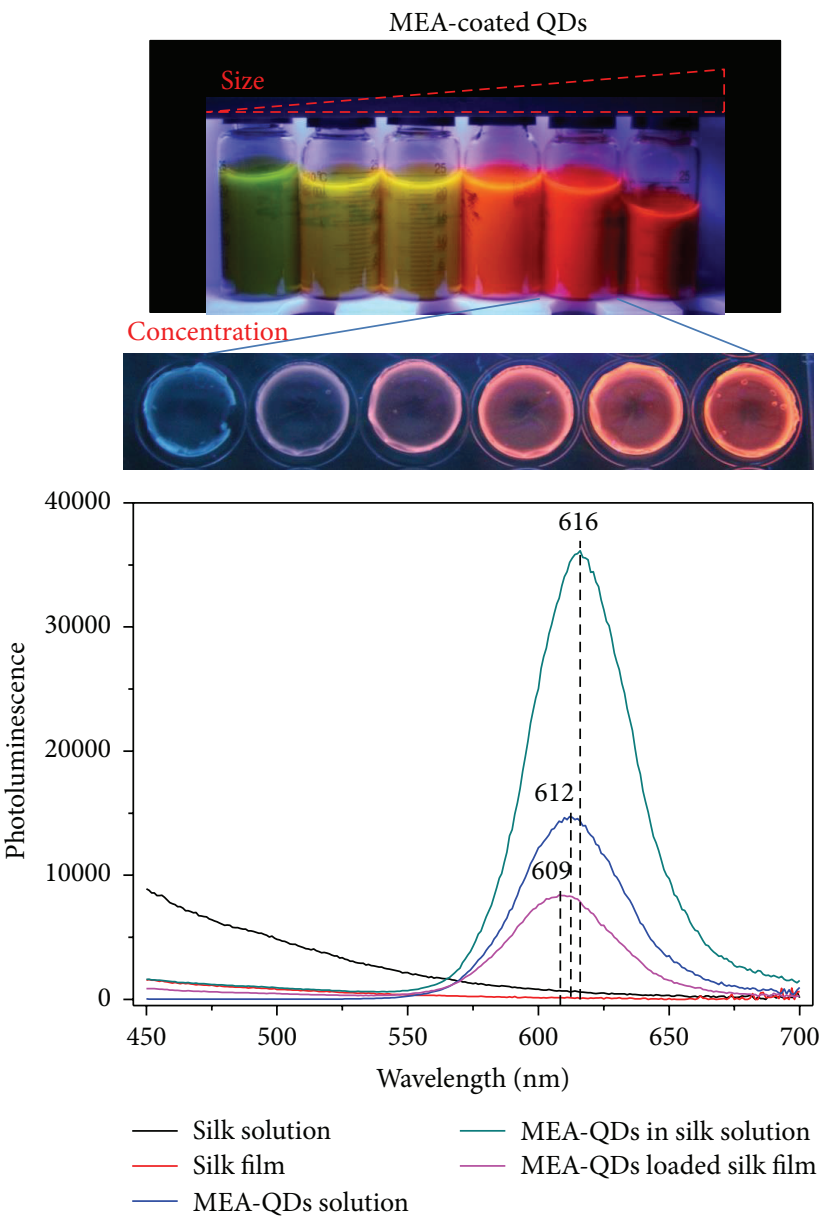

(b)

FIGURE 1: QDs-loaded silk films exposed to UV light (excitation wavelength at $365 \mathrm{~nm}$ ). Upper photographs showed as-prepared QDs in solution and encapsulated in silk/glycerol (weight ratio 3/1) films. Lower spectra showed the photoluminescence of as-prepared QDs in water, 4\% silk (SF) solution, and silk/glycerol (SF) film, using QDs-free SF solution and SF film as controls. (a) MPA-capped CdTe-QDs. The mass of silk was $12 \mathrm{mg}$ in each film, and the amount of QDs loaded was (from left to right) 0, 9.75, 19.5, 39, 52, and 78 $\mu \mathrm{mol}$. (b) MEA-capped CdTe-QDs. The mass of silk was $12 \mathrm{mg}$ in each film, and the amount of QDs loaded was (from left to right) $0,15.75,31.5,63,84$, and 126 $\mu \mathrm{mol}$.

the air (methanol treatment). All these treatments induced silk beta-sheet structure formation to some extents, thus making silk film water insoluble. The addition of glycerol (>30\% silk mass) conferred water-insolubility to silk films and also made the films more flexible and smooth than methanol-treated and water-annealed films [26].

2.2.4. Photoluminescence Measurement and Photography. Photoluminescence spectra were recorded using a synergy $\mathrm{H} 1$ microplate reader (Bio-Tek, USA) at excitation wavelength of $380 \mathrm{~nm}$ and emission wavelength of 450-700 nm, in black 96well plates (Conning). If not specifically stated, the top probe was set with a vertical offset of $7.00 \mathrm{~mm}$ and sensitivity of 100 . Photographs were taken by a Cannon 60D digital camera.

\subsubsection{Silk Structure Determination by Attenuated Total} Reflectance Fourier Transform Infrared Spectroscopy (ATRFTIR). Silk films with and without encapsulation of QDs were loaded into an ATR-FTIR instrument (Nicolet 5700,
Thermo Fisher Scientific Inc., America) with resolution of $4 \mathrm{~cm}^{-1}$, scanning range from 400 to $4000 \mathrm{~cm}^{-1}$, and 64 scans in total. The peaks shown at $1616-1637 \mathrm{~cm}^{-1}$ and $1695-$ $1705 \mathrm{~cm}^{-1}$ were assigned to silk $\beta$-sheet structure, $1638-$ $1655 \mathrm{~cm}^{-1}$ to random coil structure, $1656-1663 \mathrm{~cm}^{-1}$ to $\alpha$ helical structure, and $1663-1695 \mathrm{~cm}^{-1}$ to $\beta$-turn structure [27]. To identify the proportion of beta-sheet structure among all secondary structures of silk, the absorbance peaks in the Amide I region (1595-1705 $\mathrm{cm}^{-1}$ ) were deconvoluted using Peakfit software with three repeats of data fitting for each sample $(N=3)$.

2.2.6. Proteolytic Degradation. The QDs-loaded silk films were treated with protease XIV, alpha-chymotrypsin, and the combination of protease XIV and alpha-chymotrypsin (1/1 weight ratio) in $0.1 \mathrm{M}$ phosphate buffer, $\mathrm{pH}$ 7.4. Silk films formed in 24 wells were immersed in $1 \mathrm{~mL}$ of freshly prepared protease solution $(300 \mu \mathrm{g} / \mathrm{mL})$. After incubation at $37^{\circ} \mathrm{C}$ for $24 \mathrm{~h}$, the solution containing noncleavable silk particles 


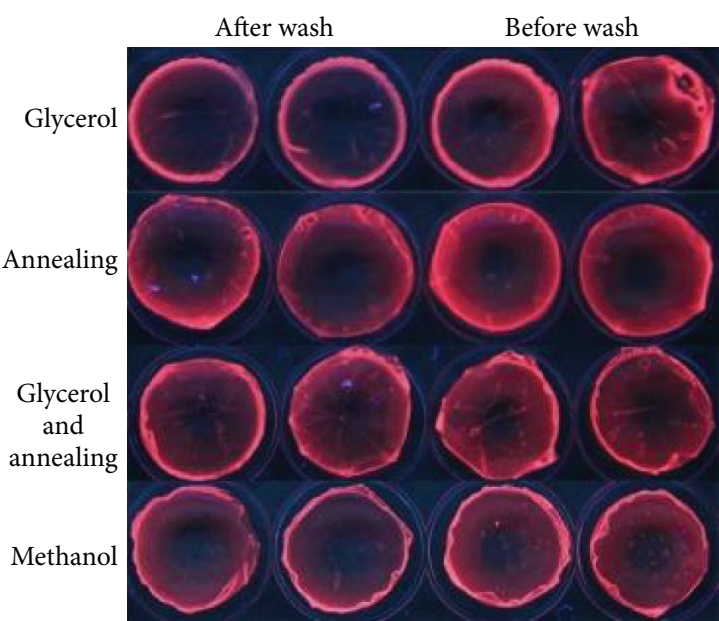

(a)

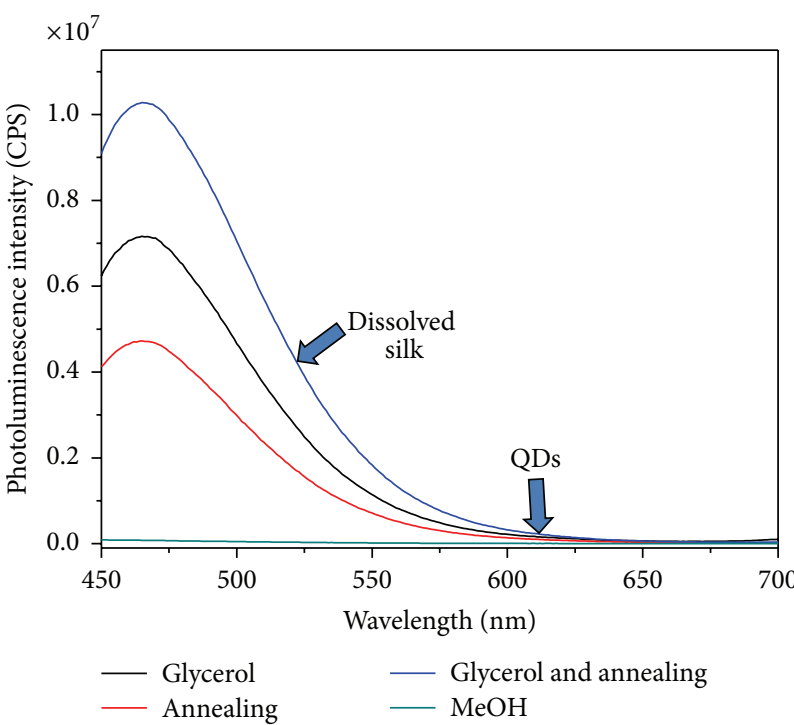

(c)

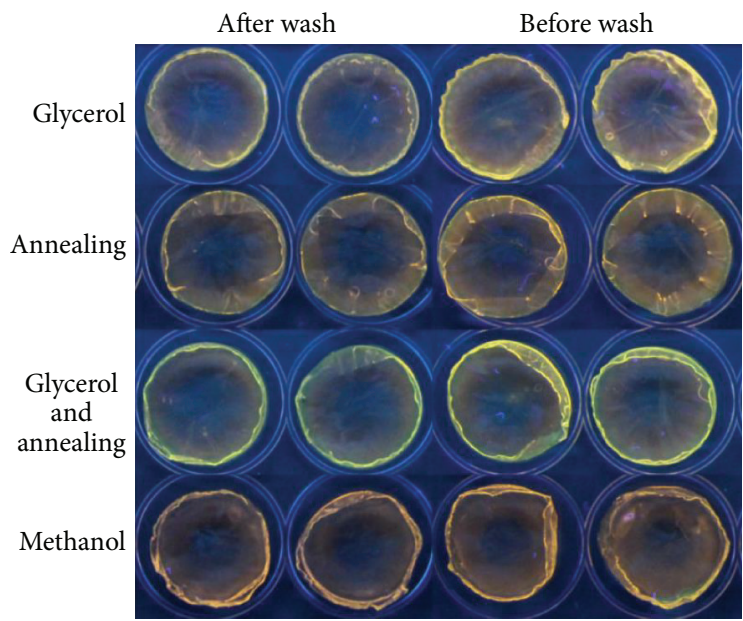

(b)

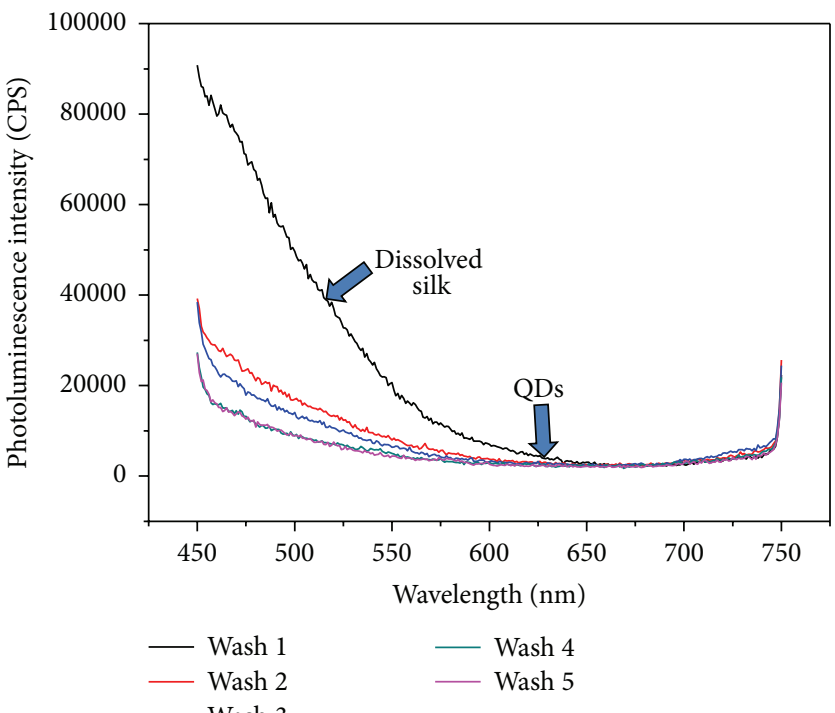

(d)

FIGURE 2: Binding of MPA-QDs and MEA-QDs to silk. (a) MPA-QDs-loaded silk films after various treatments were washed with water. (b) MEA-QDs-loaded silk films after various treatments were washed with water. (c) Photoluminescence spectra of the solutions collected from washing MPA-QDs-loaded silk films. (d) Photoluminescence spectra of five washing solutions from the methanol-treated MPA-QDsloaded silk films.

(precipitate) was removed to an empty tube. Silk films without exposure to proteases (controls), residual film debris after removal of solution, and digested solution were photographed under a UV light with excitation wavelength of $365 \mathrm{~nm}$.

2.2.7. QDs Photoluminescence Quenching by Hydrogen Peroxide $\left(\mathrm{H}_{2} \mathrm{O}_{2}\right)$. QDs-loaded silk films were prepared as described above in a 24-well plate. Each film was immersed in $900 \mu \mathrm{L}$ PBS buffer, $\mathrm{pH}$ 7.4. After incubation for $5 \mathrm{~min}, 100 \mu \mathrm{L}$ $\mathrm{H}_{2} \mathrm{O}_{2}$ solution with concentrations of $0.01,0.05,0.1,0.5,0.75$, $1,2,3,3.5,4,4.5,5,6.25,7.5,8.75,10,25,50,75$, and $100 \mathrm{mM}$ (diluted from $10 \mathrm{M} \mathrm{H}_{2} \mathrm{O}_{2}$ stock solution) was added to each well to start reaction. In the control wells $100 \mu \mathrm{L}$ ultrapure water instead of $\mathrm{H}_{2} \mathrm{O}_{2}$ was added. The plate was placed at $37^{\circ} \mathrm{C}$ and incubated for 15 min before being photographed under the UV light. The films were then removed from the wells and cut into small pieces with a defined size and weight, which were placed into black 96-well plates for photoluminescence measurement. To compare photoluminescence intensity changes in response to $\mathrm{H}_{2} \mathrm{O}_{2}$, the photoluminescence maximum for each sample was normalized by the photoluminescence intensity in the control sample. Four repeat samples $(N=4)$ were measured for each $\mathrm{H}_{2} \mathrm{O}_{2}$ concentration.

2.2.8. Statistics. Results were expressed as means \pm standard deviations. Statistical differences between samples were evaluated in SPSS (16.0) using one-way ANOVA test. Difference was considered significant when $p<0.05$. 


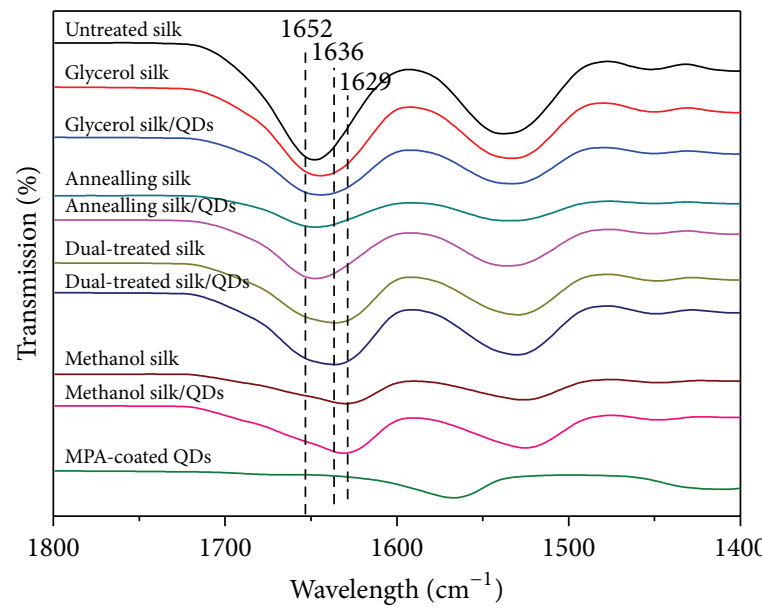

(a)

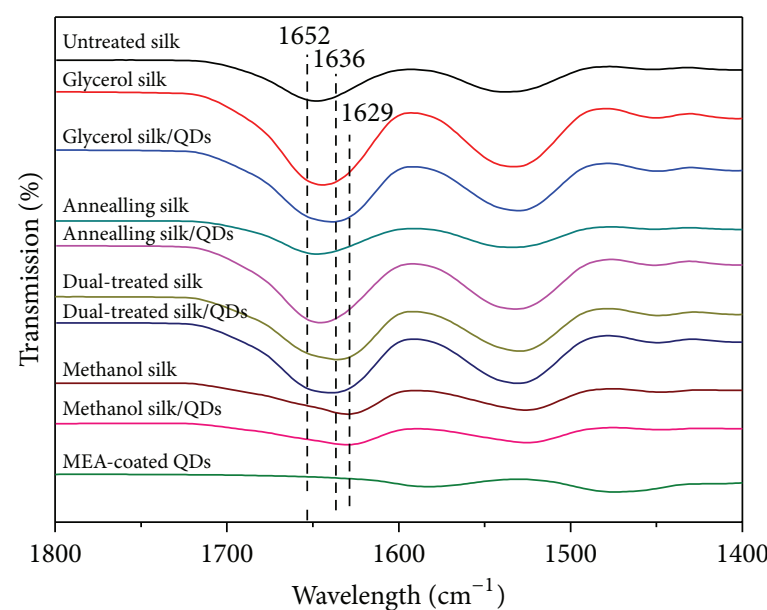

(b)

FIGURE 3: ATR-FTIR spectra of the as-prepared QDs, silk films, and QDs-loaded silk films. (a) MPA-QDs-loaded silk film. (b) MEA-QDsloaded silk film.

\section{Results and Discussion}

3.1. Physical Characterization of QDs-Loaded Silk Films. MPA- and MEA-capped QDs were synthesized as described in Section 2. Depending on the size, the fluorescence emissions of QDs were different (from green to red) (Figure 1). The red fluorescent QDs (diameter approximately $4.5 \mathrm{~nm}$ according to the literature $[24,25]$ ) were chosen for the current study. When MPA- and MEA-QDs were mixed with silk/glycerol $(3 / 1 \mathrm{w} / \mathrm{w})$ solution and the mixture was airdried in the fume hood, both MPA-silk and MEA-silk films fluoresced under UV light. Photoluminescence increased with the increase of MPA-QDs and MEA-QDs concentrations in the films (Figure 1). The films containing $39 \mu \mathrm{mol} \mathrm{MPA-}$ QDs and $63 \mu \mathrm{mol}$ MPA-QDs (the 4 th one from left to right on Figure 1) showed stronger photoluminescence than those with less QDs loading (to the left) while showing less difference from those with more QDs loading (to the right). The loading conditions were thus used for the following studies.

The emission maximum of MPA-QDs was at $635 \mathrm{~nm}$ when diluted in water and in silk solution and $631 \mathrm{~nm}$ when dried in the silk/glycerol film. The photoluminescence maximum of MEA-QDs was at 616, 612, and $610 \mathrm{~nm}$ when diluted in water, silk solution, and silk/film, respectively (Figure 1). Thus, MPA-QDs were more stable than MEAQDs in terms of photoluminescence. QDs-incorporated silk films were treated in different ways (glycerol addition, waterannealing, glycerol with water-annealing, and methanol treatment) and washed with water to determine QDs release from the films. Both MPA-QDs and MEA-QDs bound tightly to silk, as seen by almost unchanged photoluminescence from the silk films after washing (Figure 2, upper photographs) and low levels of photoluminescence in washing solution determined by photoluminescence spectroscopy (Figure 2, lower graphs). The high peaks seen in the graphs were actually from the intrinsic fluorescence from silk fibroin protein; the photoluminescence from QDs at 620-630 nm was negligible even in the first washing solution (see arrows in the graphs).
For MEA-QDs, neither the silk nor QDs photoluminescence was detectable in the washing solution. The fact that QDs can tightly bind to silk with minimal change of photoluminescence properties (intensity, emission maximum) suggests that the complications of cross-linking or crystal growth during processing can be avoided. Thus the QDs can be easily incorporated in silk materials via physical binding, facilitating the fabrication of silk-based optical sensors and other applications.

3.2. Mechanism of QDs Binding to Silk. Silk has been known to predominately consist of repeating hexapeptides GAGAGS and GAGAGY, which form crystalline beta-sheet structure in silk materials (e.g., films) after various treatments mentioned above, conferring strength and chemical and physical stabilities to silk materials [19]. Previous studies have shown that many types of molecules, from low molecular weight compounds to proteins, bind to silk, likely due to intermolecular interaction between the molecules and silk beta-sheet structure domains, along with the amphipathic characteristics $[20,21]$. The same mechanism might also apply to QDs, as the hydrophobic core of QDs might interact with the hydrophobic beta-sheet domains in silk. When the secondary structure of silk fibroin protein was examined by ATR-FTIR, it was found that all the QDs-incorporated silk films after treatments exhibited characteristic peaks at 1616$1637 \mathrm{~cm}^{-1}$ in Amide I region, corresponding to beta-sheet structure, whereas the untreated silk film (as-cast) showed a peak at $1652 \mathrm{~cm}^{-1}$, corresponding to random coil structure (Figure 3). Further calculation of beta-sheet structure content by peak deconvolution indicated that the betasheet structure content in glycerol-blended, water-annealed, and glycerol-blended/water annealed films was 20-30\% of the total structure, whereas it was $30-40 \%$ and less than $20 \%$ for the methanol-treated and untreated films, respectively (Figure 4). Compared to other treatment methods, methanol-treated films showed significantly higher 


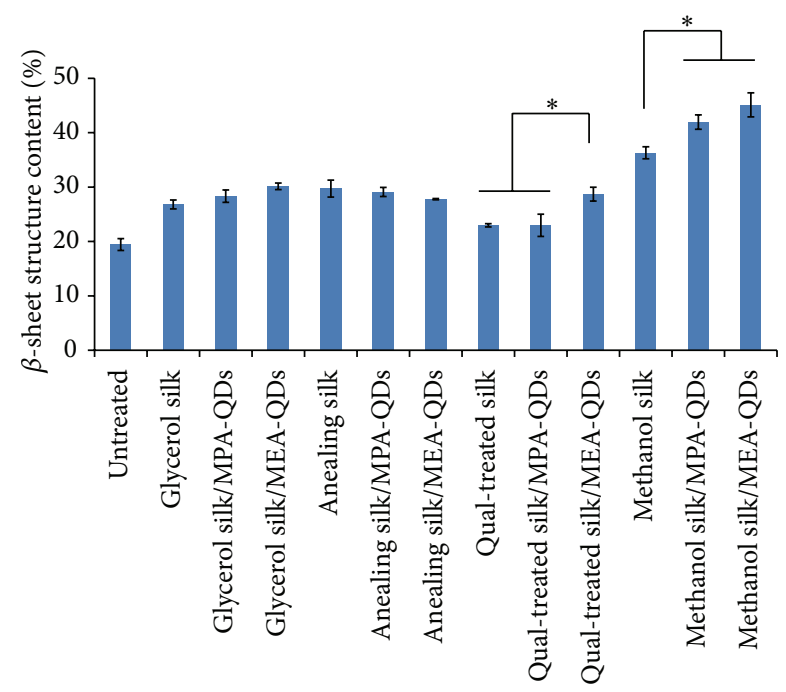

FIGURE 4: Determination of $\beta$-sheet structure contents in silk films by deconvolution of the Amide I peaks $\left(1595-1705 \mathrm{~cm}^{-1}\right.$ ) on ATRFTIR spectra. Significant difference between samples was indicated by asterisk $(p<0.05)$.

beta-sheet structure content, consistent with the literature [27]. Compared with plain silk films (no QDs), incorporation of MPA-QDs and MEA-QDs in silk films did not result in significantly more beta-sheet structures except for the glycerolblended/water-annealed MEA-QDs-incorporated silk films and methanol-treated MPA- and MEA-QDs films (Figure 4).

Silk materials could be digested by different proteases. Protease XIV cleaved both random coil and crystalline betasheet regions in silk, more efficiently for the random coil than the beta-sheet regions, while alpha-chymotrypsin only cleaved the random coil regions $[28,29]$. Upon alpha-chymotrypsin digestion, the noncleavable hydrophobic beta-sheet domains formed water-insoluble precipitates. MPA-QDsincorporated glycerol-blended silk films were used in this protease study. After alpha-chymotrypsin digestion, the solution was removed to an empty tube, in which the noncleavable silk particles precipitated and strongly photoluminesced at the bottom of the tube, while the supernatant was not photoluminescent, indicating the QDs were still bound to the crystalline beta-sheets (2nd row of photos in Figure 5). In the case of protease XIV (1st row of photos in Figure 5), the photoluminescence from the precipitate was weaker compared to that of alpha-chymotrypsin, suggesting that part of the crystalline beta-sheets was digested, releasing QDs into the supernatant. When dual proteases were used, the films were completely digested, with no visible precipitate in the tube (Figure 5, 3rd row of photos). Both amorphous and crystalline beta-sheet regions of silk were digested, resulting in the release of the bound QDs in this case. The photoluminescence spectra for the supernatants were taken using a photoluminescence spectrometer. As shown in Figure 5 (lower graph), the emission maxima of the supernatants after protease digestion blue-shifted from 631 to $621 \mathrm{~nm}$. Photoluminescence intensities in the supernatants were also significantly lower than that of control QDs at the same concentration. Thus,

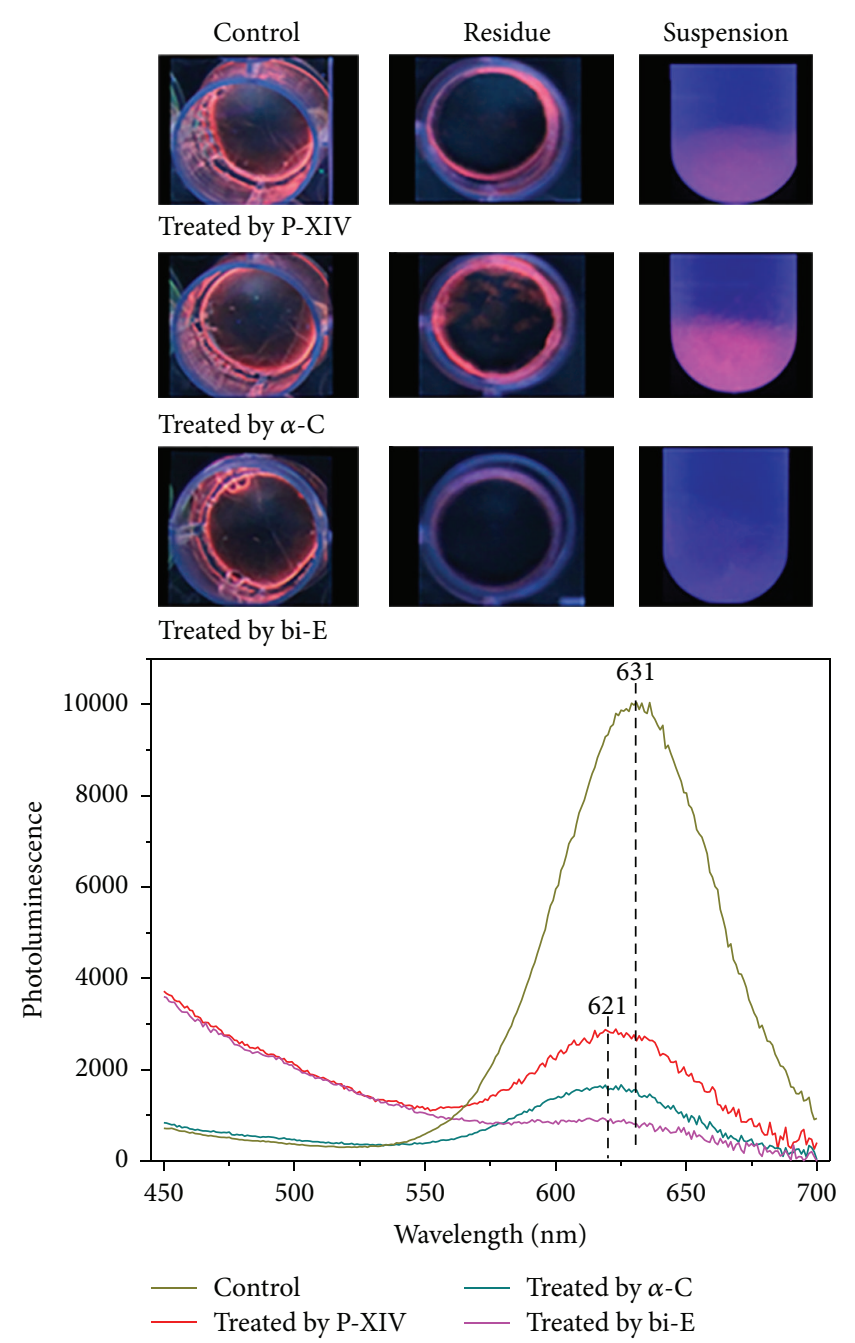

FIgURE 5: Proteolytic degradation of MPA-QDs-loaded silk films by protease XIV, alpha-chymotrypsin, and the combination of protease XIV and alpha-chymotrypsin. Upper photographs from the left to the right: control (no proteases), residual silk films after removal of digested solution, and digested solution in a tube. The samples were exposed to the UV light with an excitation wavelength at $365 \mathrm{~nm}$. Lower graph: photoluminescence spectra of the supernatants after removal of noncleavable silk precipitates at the bottom of the tubes.

protease digestion might have influenced the size and photoluminescence of QDs after they were released from the bound silk matrix. Photoluminescence intensity of the supernatant after protease XIV digestion was higher than that after alphachymotrypsin digestion, suggesting protease XIV digestion released more QDs from silk films, consistent with the photographic observations.

The mechanism underlying interaction of QDs with silk is schematically presented in Figure 6. Silk fibroin extracted from B. mori is composed of three structural components (heavy chain, light chain, and P25). The heavy chain, which is the key component to determine material properties, consists of the hydrophilic regions (nonrepetitive $\mathrm{N}$ - and C-terminus and spacer) and hydrophobic, repeating, large hexapeptide domains (GAGAGS, GAGAGY) [19]. Depending on 


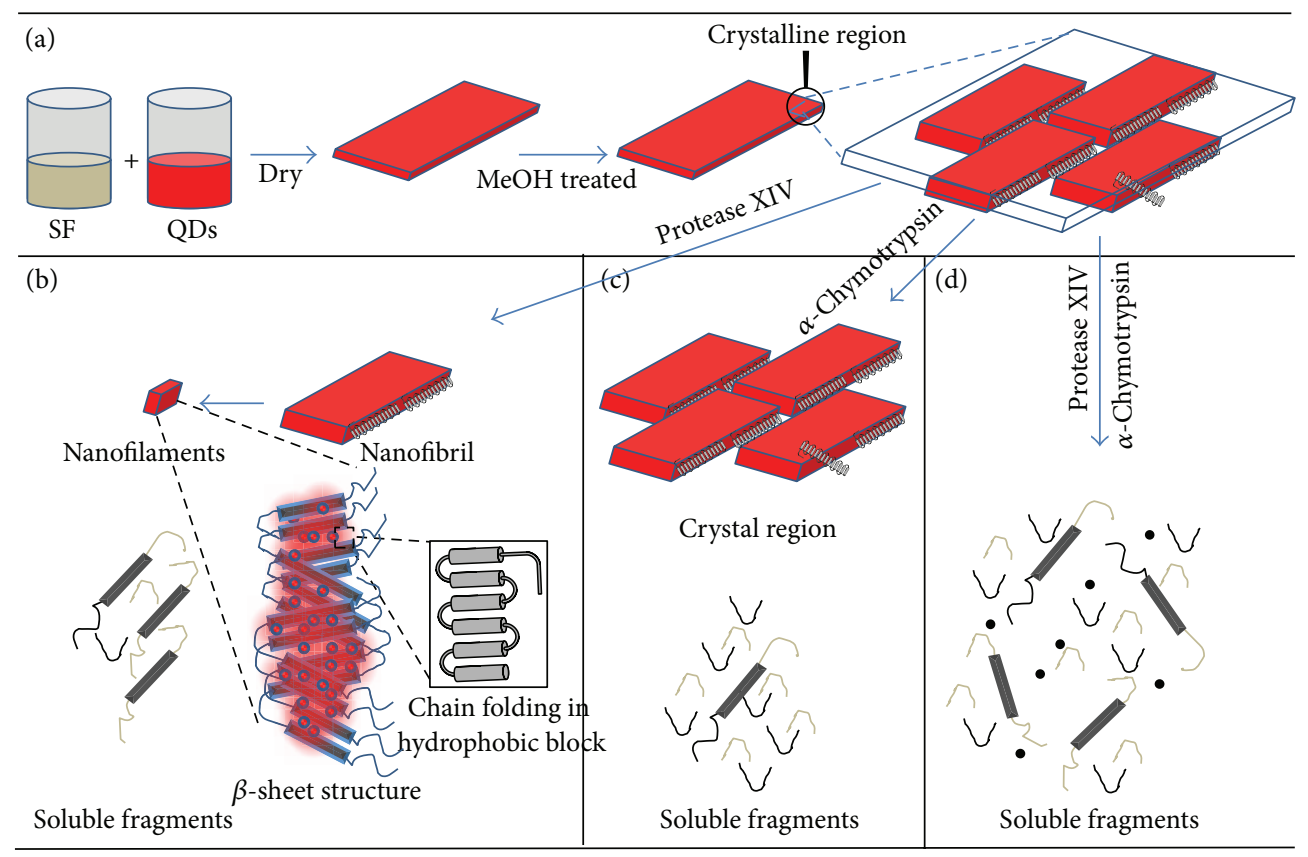

FIGURE 6: Schematic illustration of proteolytic degradation of QDs-loaded silk films. Crystalline beta-sheet regions in silk films were induced after methanol treatment (a). Silk crystalline beta-sheet regions were partially cleaved into nanofibril and nanofilaments by protease XIV (b), while they remained intact in the presence of alpha-chymotrypsin (c). The random coil regions of silk molecules were cleaved into soluble fragments by both protease XIV and alpha-chymotrypsin. Beta-sheet and random coil regions were both cleaved by the dual proteases (protease XIV and alpha-chymotrypsin), yielding more soluble fragments in solution (d).

the processing, the repetitive hexapeptide domains can form different contents of crystalline beta-sheet regions, conferring tunable strength and stability to silk biomaterials. Various types of compounds, from small molecules to peptides and proteins, can bind to silk via different mechanisms, including hydrogen bonding, electrostatic interaction, and hydrophobic interaction [20,21]. In the present study, binding of QDs to the crystalline beta-sheet regions on silk without significant change of photoluminescence properties was demonstrated. Of note, although only methanol treatment to induce silk crystalline beta-sheet structure was indicated in the process, other treatments, for example, glycerol addition, water-annealing, and combinations of both can also induce the beta-sheet structures to lesser degrees, as indicated in the ATR-FTIR study. Thus, the mechanism described should apply to all of the silk films prepared with the different treatments.

\section{3. $\mathrm{H}_{2} \mathrm{O}_{2}$-Induced Photoluminescence Quenching of QDs-Silk} Films. To demonstrate that QDs-incorporated silk films can be used for optical sensing in biomedical applications, the response of QDs photoluminescence to hydrogen peroxide $\left(\mathrm{H}_{2} \mathrm{O}_{2}\right)$ in solution was examined. MPA-QDs- and MEA-QDs-incorporated silk films with glycerol addition and methanol treatment were used in the study. $\mathrm{H}_{2} \mathrm{O}_{2}$ was added to the wells containing different films and PBS buffer, $\mathrm{pH} 7.4$, to obtain a concentration range of $0.001-$ $10 \mathrm{mM}$. The samples were photographed after reaction for $15 \mathrm{~min}$ at $37^{\circ} \mathrm{C}$ (Figures $7(\mathrm{~A})$ and $7(\mathrm{~B})$ ). Both glyceroladded and methanol-treated films showed significant photoluminescence quenching when the $\mathrm{H}_{2} \mathrm{O}_{2}$ concentration reached $0.02-0.03 \mathrm{mM}$ (2nd-3rd well in the second row). The films were subjected to quantitative photoluminescence determination (Figure $7(\mathrm{C})$ ). Compared to the initial photoluminescence $\left(0 \mathrm{mM} \mathrm{H} \mathrm{H}_{2} \mathrm{O}_{2}\right)$, the photoluminescence intensity decreased approximately $40 \%, 75 \%, 45 \%$, and 65\% for MEA-QDs/glycerol film, MPA-QDs/glycerol film, MEA-QDs/methanol film, and MPA-QDs/methanol film, respectively, when the concentration of $\mathrm{H}_{2} \mathrm{O}_{2}$ was above $0.2 \mathrm{mM}$, confirming the photographic observations. Furthermore, it seems the glycerol-added films were more sensitive than the methanol-treated films and MPA-QDs were more sensitive than MEA-QDs to the $\mathrm{H}_{2} \mathrm{O}_{2}$ quenching. For both types of films, the decrease of photoluminescence plateaued after $\mathrm{H}_{2} \mathrm{O}_{2}$ concentration reached $2 \mathrm{mM}$ (Figure $7(\mathrm{C})$ ), indicating that all exposed QDs had been quenched at this point. Unquenchable QDs might be those deeply embedded in the silk matrix, likely the hydrophobic beta-sheet stacks, which could not be penetrated by the $\mathrm{H}_{2} \mathrm{O}_{2}$ molecules.

$\mathrm{H}_{2} \mathrm{O}_{2}$ is generated during the glucose oxidase (GOD) catalyzed reaction of $\beta \mathrm{D}$-glucose and oxygen and has been used as an indicator for glucose level in diabetes [30]. $\mathrm{H}_{2} \mathrm{O}_{2}$ can also be generated during pathogenic biological processes, for example, cancer, stroke, renal failure, anathema, and chronic obstructive pulmonary disease (COPD), and thus has been used as an indicator for in vitro and in vivo diagnosis [31]. Due to its low concentrations $(<0.1 \mu \mathrm{m}), \mathrm{H}_{2} \mathrm{O}_{2}$ had to be condensed from the exhaled breath prior to electrochemical or colorimetrical assays. Recent progress on nanomaterials has significantly improved the sensitivity and accuracy of $\mathrm{H}_{2} \mathrm{O}_{2}$ 


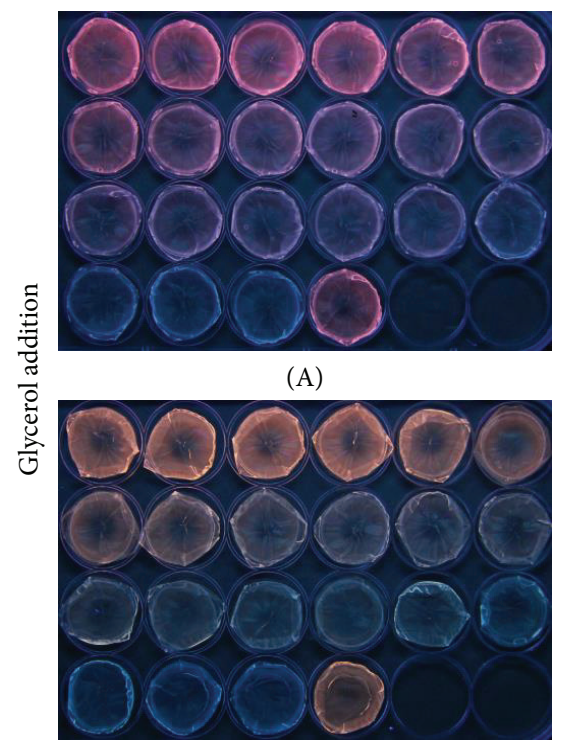

(B)

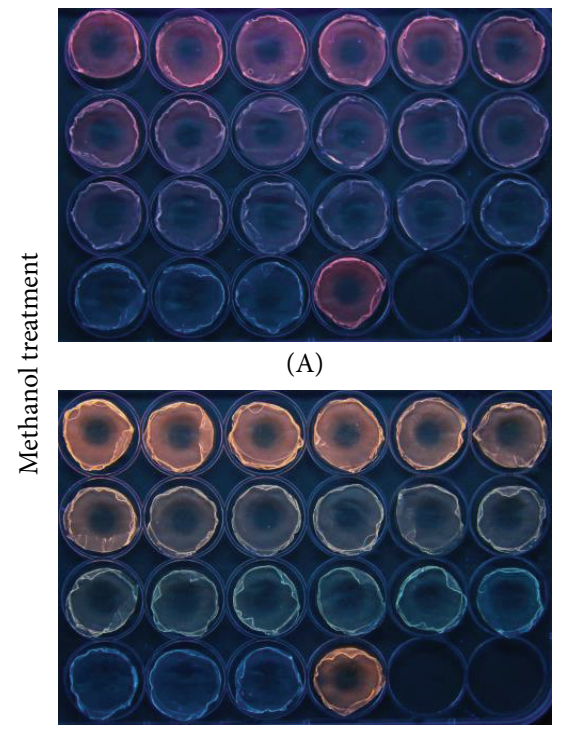

(B)

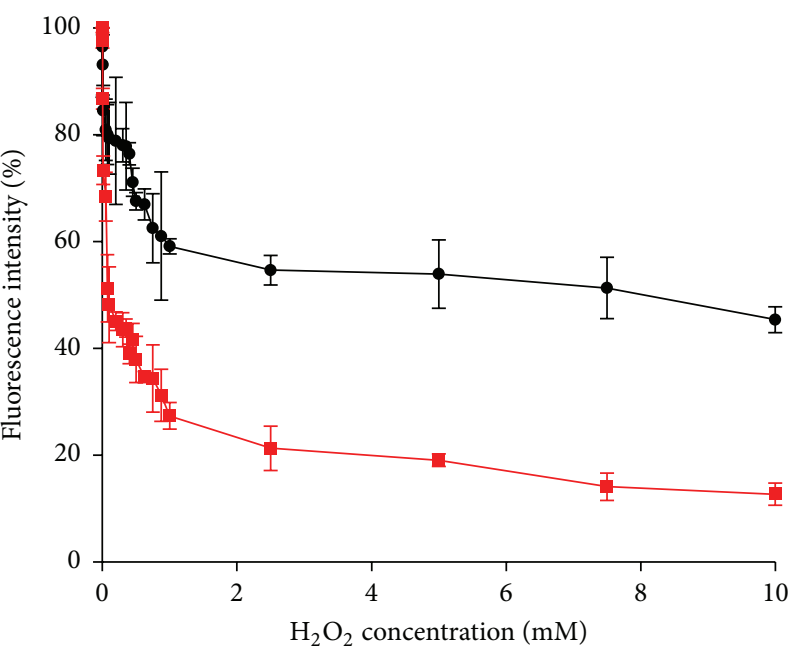

- MEA-QDs

- - MPA-QDs

(C)

(a)

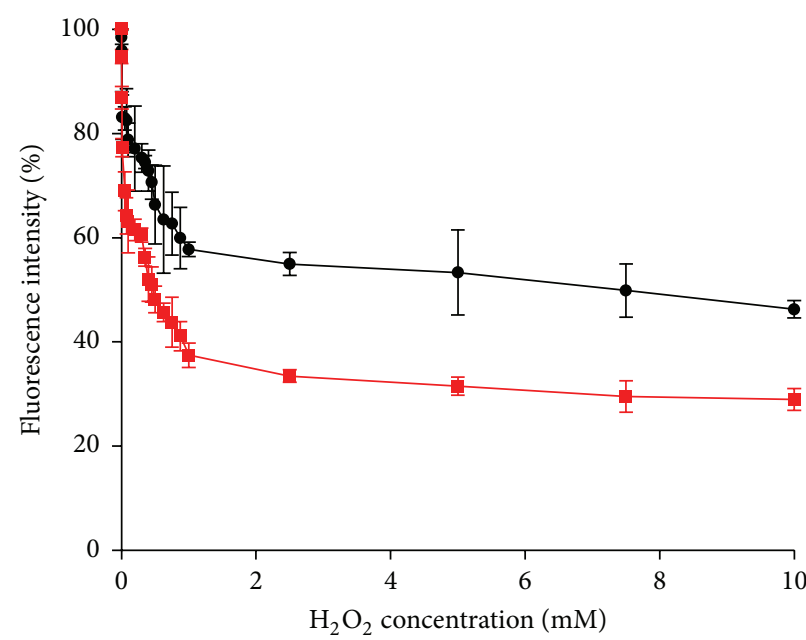

MEA-QDs

MPA-QDs

(C)

(b)

FIGURE 7: Change of photoluminescence intensities in QDs-loaded silk films in response to $\mathrm{H}_{2} \mathrm{O}_{2}$. (a) shows glycerol-added silk films. (b) shows methanol-treated silk films. (A) MPA-QDs-loaded silk films. (B) MEA-QDs-loaded silk films. $\mathrm{H}_{2} \mathrm{O}_{2}$ concentrations in the samples (from left to right): $0.0,0.001,0.005,0.01,0.05$, and $0.075 \mathrm{mM}$ on the 1 st row; $0.1,0.2,0.3,0.35,0.4$, and $0.45 \mathrm{mM}$ on the $2 \mathrm{nd}$ row; $0.5,0.625$, $0.75,0.875,1.0$, and $2.5 \mathrm{mM}$ on the $3 \mathrm{rd}$ row; $5.0,7.5,10.0$, and $0.0 \mathrm{mM}$ on the 4 th row. (C) Photoluminescence intensity changes as a function of $\mathrm{H}_{2} \mathrm{O}_{2}$ concentrations in solution.

detection [32]. The present study provides another option for fabricating $\mathrm{H}_{2} \mathrm{O}_{2}$ detection devices using QDs-incorporated silk materials. Due to the natural sourcing and "green" processing of silk materials, along with the strong binding of QDs to silk, QDs-incorporated silk-based optical sensors could find broad applications in biomedical and environment applications. Except for optical sensing, the physical incorporation of QDs can also be used for tracking degradation and thus the durability of silk-based biomaterials after implantation or injection in vivo.

\section{Conclusions}

MPA-capped and MEA-capped QDs were synthesized and physically incorporated in silk films that were treated in different ways to induce crystalline beta-sheet structure. 
Compared to MEA-QDs, MPA-QDs showed longer emission wavelength $(>630 \mathrm{~nm})$ and more stable photoluminescence properties after incorporation in silk films. Both types of QDs bound to silk tightly, with negligible amounts of QDs being extracted from the films. Protease digestion using protease XIV and alpha-chymotrypsin suggested that the QDs bound to hydrophobic beta-sheet domains preferentially in the silk matrix. The tightly bound QDs in both glycerol-added and methanol-treated silk films sensed $\mathrm{H}_{2} \mathrm{O}_{2}$ concentration changes in solution, with a critical concentration range of 0.2-0.3 mM, suggesting the QDs-incorporated silk materials can be used for fabricating optical sensors for diagnostic applications.

\section{Conflict of Interests}

The authors declare that there is no conflict of interests regarding the publication of this paper.

\section{Authors' Contribution}

Disi Lu and Zhaozhu Zheng contributed equally to this work and should be considered co-first authors.

\section{Acknowledgments}

This work was supported by Natural Science Foundation of China Grant (Project no. 51273138), Start-Up Fund of Soochow University (Project no. 14317432), Natural Science Foundation of Suzhou City Jiangsu Province China (Grant no. SYN201403), and Postdoctoral Science Foundation of Jiangsu Province China.

\section{References}

[1] C. J. Murphy, "Optical sensing with quantum dots," Analytical Chemistry, vol. 74, no. 19, pp. 520A-526A, 2002.

[2] D. Zimnitsky, C. Jiang, J. Xu, Z. Lin, and V. V. Tsukruk, "Substrate- and time-dependent photoluminescence of quantum dots inside the ultrathin polymer LbL film," Langmuir, vol. 23, no. 8, pp. 4509-4515, 2007.

[3] R. Gill, L. Bahshi, R. Freeman, and I. Willner, "Optical detection of glucose and acetylcholine esterase inhibitors by $\mathrm{H}_{2} \mathrm{O}_{2}$-sensitive CdSe/ZnS quantum dots," Angewandte Chemie International Edition, vol. 47, no. 9, pp. 1676-1679, 2008.

[4] H. Kuang, Y. Zhao, W. Ma, L. Xu, L. Wang, and C. Xu, "Recent developments in analytical applications of quantum dots," TrAC Trends in Analytical Chemistry, vol. 30, no. 10, pp. 1620-1636, 2011.

[5] Q. Ma and X. Su, "Recent advances and applications in QDsbased sensors," Analyst, vol. 136, no. 23, pp. 4883-4893, 2011.

[6] Y. Li, B. Li, and J. Zhang, " $\mathrm{H}_{2} \mathrm{O}_{2}$ - and $\mathrm{pH}$-sensitive CdTe quantum dots as fluorescence probes for the detection of glucose," Luminescence, vol. 28, no. 5, pp. 667-672, 2013.

[7] A. D. Saran, M. M. Sadawana, R. Srivastava, and J. R. Bellare, "An optimized quantum dot-ligand system for biosensing applications: evaluation as a glucose biosensor," Colloids and Surfaces A: Physicochemical and Engineering Aspects, vol. 384, no. 1-3, pp. 393-400, 2011.
[8] Y. Chong, Y. Ma, H. Shen et al., "The in vitro and in vivo toxicity of graphene quantum dots," Biomaterials, vol. 35, no. 19, pp. 5041-5048, 2014.

[9] C. Luccardini, C. Tribet, F. Vial, V. Marchi-Artzner, and M. Dahan, "Size, charge, and interactions with giant lipid vesicles of quantum dots coated with an amphiphilic macromolecule," Langmuir, vol. 22, no. 5, pp. 2304-2310, 2006.

[10] S. Ludwigs, A. Böker, A. Voronov, N. Rehse, R. Magerle, and G. Krausch, "Self-assembly of functional nanostructures from ABC triblock copolymers," Nature Materials, vol. 2, no. 11, pp. 744-747, 2003.

[11] T. J. Reece, S. Ducharme, A. V. Sorokin, and M. Poulsen, "Nonvolatile memory element based on a ferroelectric polymer Langmuir-Blodgett film," Applied Physics Letters, vol. 82, no. 1, pp. 142-144, 2003.

[12] S. Cho, J. Kwag, S. Jeong, Y. Baek, and S. Kim, "Highly fluorescent and stable quantum dot-polymer-layered double hydroxide composites," Chemistry of Materials, vol. 25, no. 7, pp. 10711077, 2013.

[13] B. Dubertret, P. Skourides, D. J. Norris, V. Noireaux, A. H. Brivanlou, and A. Libchaber, "In vivo imaging of quantum dots encapsulated in phospholipid micelles," Science, vol. 298, no. 5599, pp. 1759-1762, 2002.

[14] F. Osaki, T. Kanamori, S. Sando, T. Sera, and Y. Aoyama, "A quantum dot conjugated sugar ball and its cellular uptake. On the size effects of endocytosis in the subviral region," Journal of the American Chemical Society, vol. 126, no. 21, pp. 6520-6521, 2004.

[15] T. Pellegrino, L. Manna, S. Kudera et al., "Hydrophobic nanocrystals coated with an amphiphilic polymer shell: a general route to water soluble nanocrystals," Nano Letters, vol. 4, no. 4, pp. 703-707, 2004.

[16] H. Ismaili, F. Lagugné-Labarthet, and M. S. Workentin, "Covalently assembled gold nanoparticle-carbon nanotube hybrids via a photoinitiated carbene addition reaction," Chemistry of Materials, vol. 23, no. 6, pp. 1519-1525, 2011.

[17] B. R. Venugopal, N. Ravishankar, C. R. Perrey, C. Shivakumara, and M. Rajamathi, "Layered double hydroxide-CdSe quantum dot composites through colloidal processing: effect of host matrix-nanoparticle interaction on optical behavior," The Journal of Physical Chemistry B, vol. 110, no. 2, pp. 772-776, 2006.

[18] H.-B. Yao, L.-B. Mao, Y.-X. Yan, H.-P. Cong, X. Lei, and S.-H. Yu, "Gold nanoparticle functionalized artificial nacre: facile in situ growth of nanoparticles on montmorillonite nanosheets, selfassembly, and their multiple properties," ACS Nano, vol. 6, no. 9, pp. 8250-8260, 2012.

[19] F. Teulé, Y.-G. Miao, B.-H. Sohn et al., "Silkworms transformed with chimeric silkworm/spider silk genes spin composite silk fibers with improved mechanical properties," Proceedings of the National Academy of Sciences of the United States of America, vol. 109, no. 3, pp. 923-928, 2012.

[20] E. M. Pritchard, P. B. Dennis, F. Omenetto, R. R. Naik, and D. L. Kaplan, "Review: physical and chemical aspects of stabilization of compounds in silk," Biopolymers, vol. 97, no. 6, pp. 479-498, 2012.

[21] J. G. Hardy, A. Leal-Egaña, and T. R. Scheibel, "Engineered spider silk protein-based composites for drug delivery," Macromolecular Bioscience, vol. 13, no. 10, pp. 1431-1437, 2013.

[22] D. N. Rockwood, R. C. Preda, T. Yücel, X. Wang, M. L. Lovett, and D. L. Kaplan, "Materials fabrication from Bombyx mori silk fibroin," Nature Protocols, vol. 6, no. 10, pp. 1612-1631, 2011. 
[23] H. Tao, D. L. Kaplan, and F. G. Omenetto, "Silk materials-a road to sustainable high technology," Advanced Materials, vol. 24, no. 21, pp. 2824-2837, 2012.

[24] N. Gaponik, D. V. Talapin, A. L. Rogach et al., "Thiol-capping of CDTe nanocrystals: an alternative to organometallic synthetic routes," The Journal of Physical Chemistry B, vol. 106, no. 29, pp. 7177-7185, 2002.

[25] S. Kaniyankandy, S. Rawalekar, S. Verma, D. K. Palit, and H. N. Ghosh, "Charge carrier dynamics in thiol capped CdTe quantum dots," Physical Chemistry Chemical Physics, vol. 12, no. 16, pp. 4210-4216, 2010.

[26] S. Lu, X. Wang, Q. Lu et al., "Insoluble and flexible silk films containing glycerol," Biomacromolecules, vol. 11, no. 1, pp. 143150, 2010.

[27] X. Hu, D. Kaplan, and P. Cebe, "Determining beta-sheet crystallinity in fibrous proteins by thermal analysis and infrared spectroscopy," Macromolecules, vol. 39, no. 18, pp. 6161-6170, 2006.

[28] J. Brown, C. L. Lu, J. Coburn, and D. L. Kaplan, "Impact of silk biomaterial structure on proteolysis," Acta Biomaterialia, vol. 11, pp. 212-221, 2015.

[29] Z. Dong, P. Zhao, C. Wang et al., "Comparative proteomics reveal diverse functions and dynamic changes of Bombyx mori silk proteins spun from different development stages," Journal of Proteome Research, vol. 12, no. 11, pp. 5213-5222, 2013.

[30] E. Bakker, "Electrochemical sensors," Analytical Chemistry, vol. 76, no. 12, pp. 3285-3298, 2004.

[31] R. Stolarek, P. Bialasiewicz, M. Krol, and D. Nowak, "Breath analysis of hydrogen peroxide as a diagnostic tool," Clinica Chimica Acta, vol. 411, no. 23-24, pp. 1849-1861, 2010.

[32] Y. Y. Broza and H. Haick, "Nanomaterial-based sensors for detection of disease by volatile organic compounds," Nanomedicine, vol. 8, no. 5, pp. 785-806, 2013. 

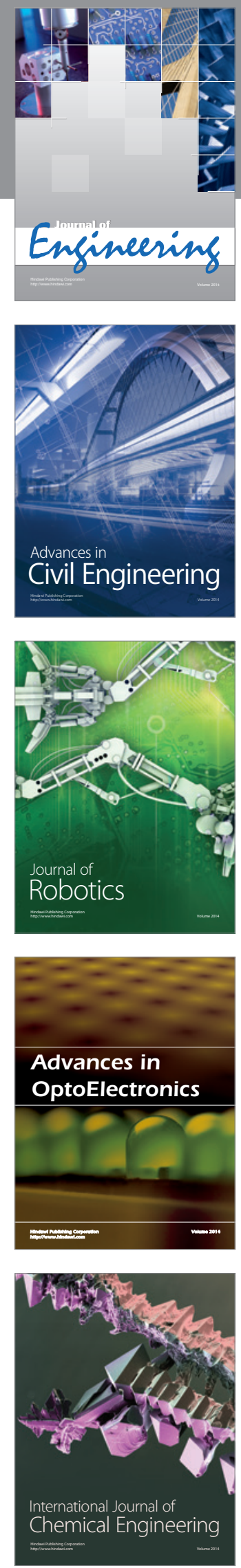

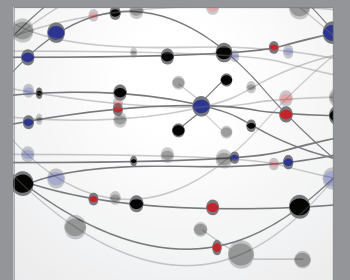

The Scientific World Journal
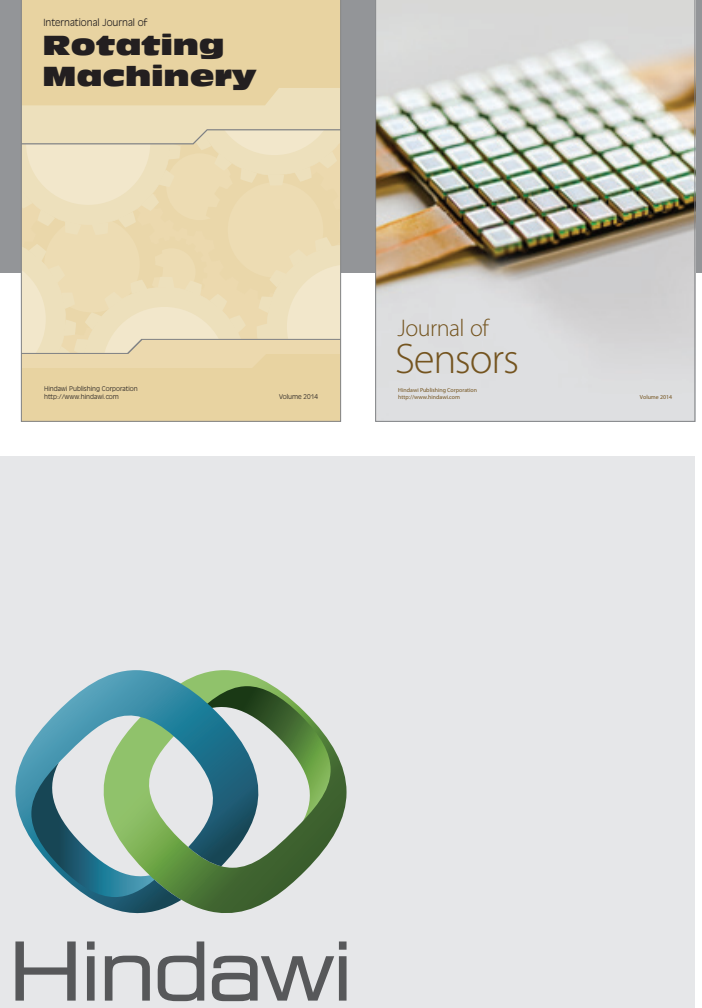

Submit your manuscripts at http://www.hindawi.com
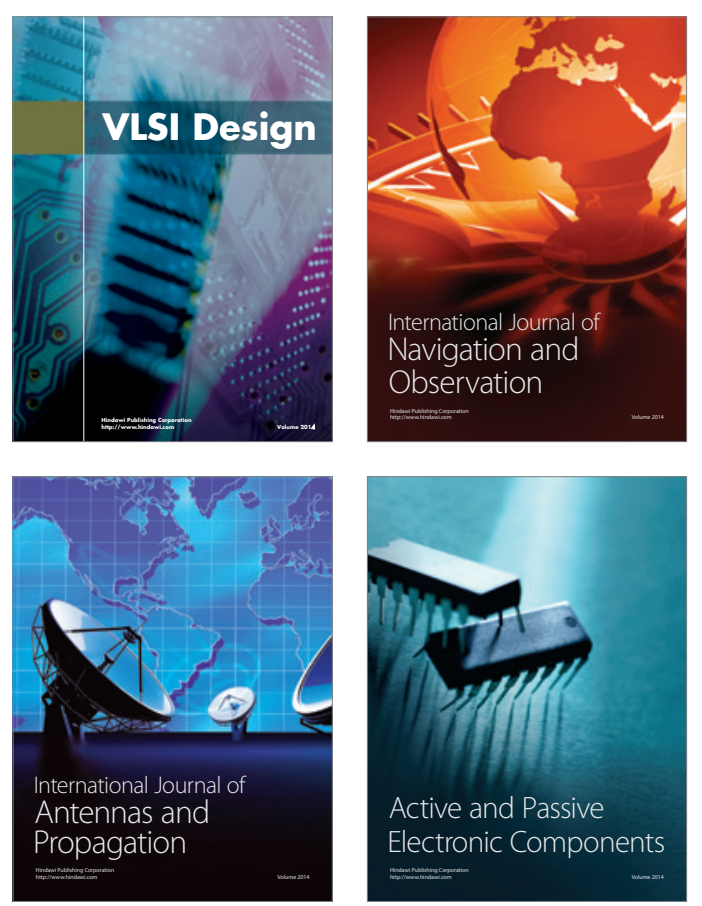
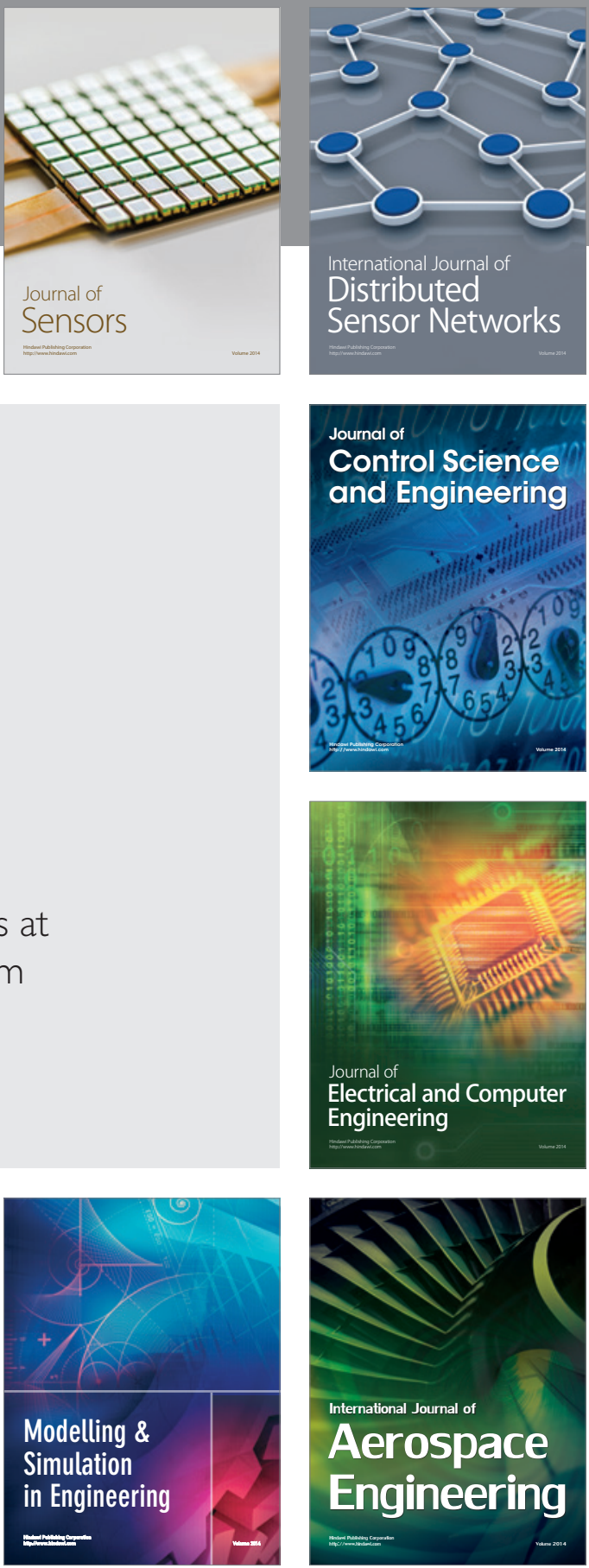

Journal of

Control Science

and Engineering
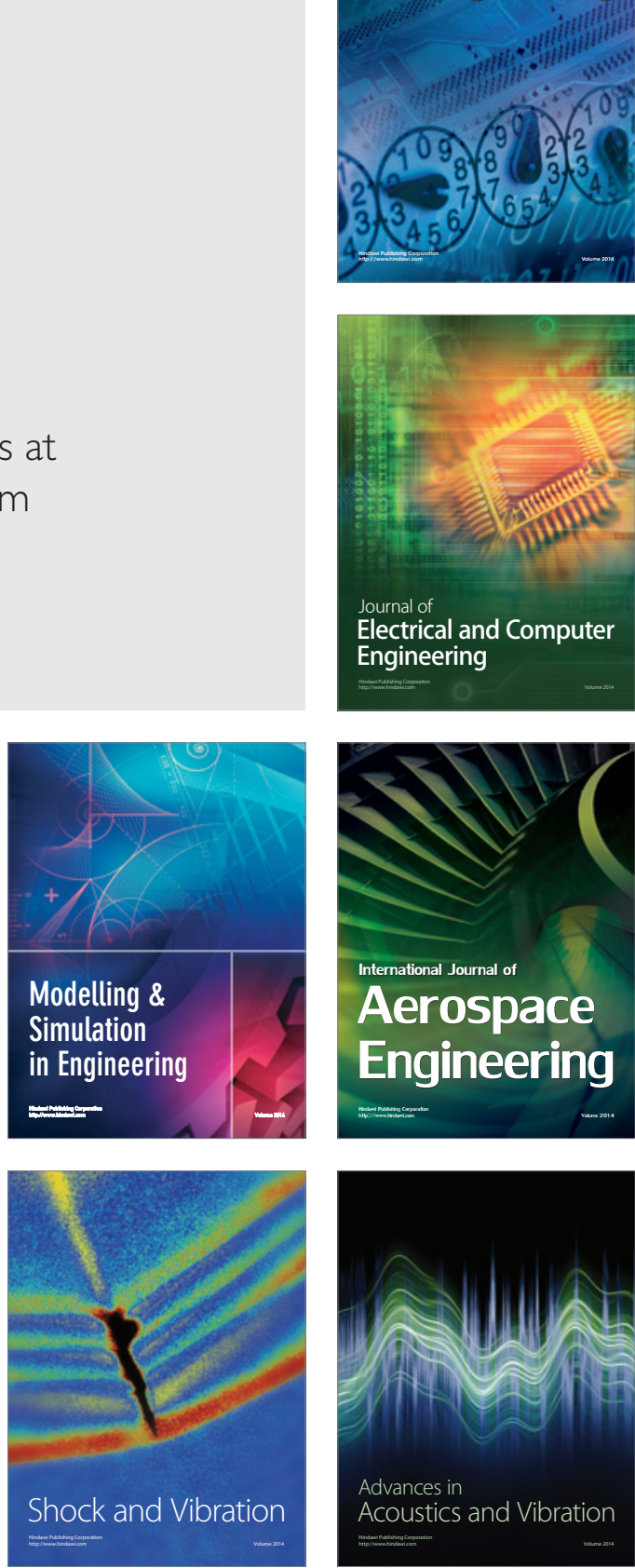